\title{
CARACTERIZAÇÃO DOS RESÍDUOS QUÍMICOS DE UM LABORATÓRIO DE ANÁLISES FÍSICO-QUÍMICAS E MICROBIOLÓGICAS DE ÁGUAS E EFLUENTES
}

\author{
Carlos Enrique de M. Jerônimo ${ }^{1}$, Henio Normando de S. Melo² \\ ${ }^{1}$ Doutorado em Engenharia Ambiental pela UFRN. Mestre em Engenharia Química. Engenheiro Especialista em \\ Processamento de Petróleo pela UERJ e Engenheiro de Processamento da Petrobrás. Engenheiro Químico pela UFRN. \\ R. Prof. Gerson Dumaresq, 259, Capim Macio, Natal - RN. E-mail:c_enrique@hotmail.com \\ ${ }^{2}$ Professor Doutor do Departamento de Engenharia Química da UFRN.
}

\section{RESUMO}

Os resíduos laboratoriais representam, atualmente, mais uma classe de materiais que vêm contribuindo com a poluição ambiental, como também, colocando em risco a saúde dos profissionais que os manuseiam e/ou mantêm contato rotineiro de outras maneiras. Diante dessa situação, as Universidades se enquadram como as principais responsáveis pelo problema, pois normalmente apresentam um grande número de laboratórios, com resíduos característicos, muitas vezes com propriedades desconhecidas e alto grau de insalubridade. Além do que, não há pressão por parte dos órgãos fiscalizadores para que estes procurem formas ambientalmente corretas de tratar ou destinar os seus resíduos. Por essas razões, esse trabalho tem como objetivo principal a caracterização dos resíduos de um Laboratório de Engenharia Ambiental e Controle de Qualidade - LEACQ - de uma instituição de ensino superior do nordeste brasileiro, tendo em vista, o futuro reaproveitamento desses materiais a partir de sua recuperação ou o descarte ambientalmente correto. Para tanto se realizou uma estimativa dos resíduos em termos qualitativos e quantitativos, através da listagem das principais análises realizadas no laboratório, assim como de sua periodicidade de execução e de seus principais reagentes. Nessa estimativa considerou-se a quantidade dos reagentes puros, desconsiderando a água de diluição. Foi adotada uma classificação para os diversos resíduos, sendo: (A) Compostos sulfonados; (B) Compostos Clorados ou Nitrogenados; (C) Compostos com Metais Pesados; (D) Compostos Orgânicos e (E) Outros. Dentro dessa classificação subdividiu-se os resíduos sólidos e os líquidos. Dos resultados obtidos com a estimativa qualitativa e quantitativa dos resíduos do LEACQ, conclui-se que a classe majoritária, em termos de contaminação é a classe $A$, principalmente pela presença de ácido sulfúrico, reagente usado em grande escala. Entretanto, a grande preocupação é a presença de metais pesados - Classe $C$ - que, mesmo em pequenas quantidades, acarretam problemas em razão do acúmulo na cadeia trófica. Porém, para complementar esse trabalho se faz necessário realizar algumas análises laboratoriais para se confrontar os resultados estimados com os experimentais.

PALAVRAS-CHAVES: Resíduos Laboratoriais; Perigosos; Químicos

\section{INTRODUÇÃO}

Cada vez mais, pode-se observar uma nova tendência de pensamento voltado para os princípios de sustentabilidade integrando os vários segmentos da sociedade, ou seja, como definiu Pedrozo \& Philippi Jr (1999): “O desafio da sociedade moderna é crescer economicamente e de 


\section{REGEIATFM \\ JERÔNIMO \& MELO, v(7), no 7, p. 1520-1526, MAR-AGO, 2012.}

Rev. Elet. em Gestão, Educação e Tecnologia Ambiental (e-ISSN: 2236-1170)

forma sustentável integrando os vários segmentos sociais e suas necessidades à qualidade dos produtos, sem prejuízo da qualidade ambiental".

Desse modo, o que vem sendo visto é que locais antes considerados não causadores de impactos ambientais passaram a ser melhor estudados e incluídos dentro de uma vista gama de setores geradores de atividades e serviços que afetam as características do meio ambiente de forma mais amena, porém, que a longo prazo podem ser responsáveis por efeitos altamente prejudiciais a qualidade ambiental.

As atividades que vêm sendo incentivadas e que aos poucos também cobradas pelos órgãos públicos estão relacionadas à quantificação dos resíduos, à classificação dos materiais, ao controle da geração e à disposição adequada.

Diante dessa situação os resíduos químicos laboratoriais representam, atualmente, mais uma classe de materiais que vêm contribuindo com a poluição ambiental, como também colocando em risco a saúde dos profissionais que os manuseiam e/ou mantêm contanto rotineiro de outras maneiras.

Esses resíduos são considerados de caráter especial, principalmente pelo grau de periculosidade e diversidade de composição apresentada, podendo-se encontrar substâncias classificadas como tóxicas, explosivas, inflamáveis, corrosivas, reativas etc.

Dentre os diversos tipos de instituições que geram resíduos laboratoriais as universidades e institutos de pesquisas se enquadram como uma das principais responsáveis pelo problema, pois normalmente apresentam um grande número de laboratórios, com determinações analíticas diversas, resíduos característicos (muitas vezes com propriedades desconhecidas) e alto grau de insalubridade. Além do que, não há pressão por parte dos órgãos fiscalizadores para que essas entidades procurem formas ambientalmente corretas de tratar ou destinar os seus resíduos.

Por essas razões, esse trabalho tem como objetivo a caracterização dos resíduos de um laboratório de Engenharia Ambiental e Controle de Qualidade - LEACQ - de uma instituição de ensino superior do nordeste brasileiro, tendo em vista, o futuro reaproveitamento desses materiais a partir de sua recuperação ou o descarte ambientalmente correto.

\section{DESCRIÇÃO DO LOCAL ESTUDADO}

O LEACQ é responsável pela realização de uma vasta gama de determinações analíticas na área de meio ambiente e controle de qualidade. Nesse laboratório são avaliadas as principais características físico-químicas e microbiológicas de rios, poços, lagoas, estações de tratamento de efluentes e de variados tipos de solos.

Desse modo, o número de substâncias envolvidas nessas determinações é muito grande, aumentando ainda mais a necessidade de um tratamento específico para cada tipo de resíduo.

Entre as análises realizadas podemos citar:

$>$ Microbiológicos (coliformes totais e fecais);

$>$ Sólidos (totais, dissolvidos e suspensos);

$>$ Demanda Química de Oxigênio (DQO);

$>$ Carbono Orgânico Total (COT);

$>$ Metais Pesados;

Nitrogênio (nitratos, nitritos etc);

$>$ Enxofre;

$>$ Fósforo; 


\section{REGETHFSM

Rev. Elet. em Gestão, Educação e Tecnologia Ambiental (e-ISSN: 2236-1170)

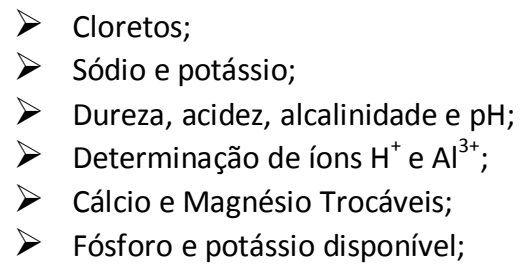

\section{METODOLOGIA}

Para a caracterização desejada, realizou-se uma estimativa dos resíduos em termos qualitativos e quantitativos, através da listagem das principais análises realizadas no laboratório, assim como a junção temporal a qual se realizam essas análises e os seus principais reagentes.

Através de uma varredura pela literatura, em busca de trabalhos que desenvolveram metodologias similares, definiu-se a classificação a ser adotada. Desse modo, dividiu-se o resíduo da seguinte maneira:
(A) Compostos Sulfonados;
(B) Compostos Clorados ou Nitrogenados;
(C) Compostos com Metais Pesados;
(D) Compostos Orgânicos;
(E) Outros

Dentro dessa classificação subdividiu-se os resíduos em sólidos e líquidos.

Nessa estimativa considerou-se a quantidade dos reagentes puros para se determinar os resíduos de cada espécie. Para tanto, nos cálculos realizados desconsiderou-se a água de diluição e levou-se em conta também a questão da pureza dos reagentes. Através de cálculos estequiométricos e balanços parciais de massa foi possível a determinação do consumo exato dos materiais.

\section{RESULTADOS E DISCUSSÕES}

A análise dos resultados tem inicio com a descrição dos tipos de reagentes utilizados no laboratório, para suas respectivas análises e quantidades necessárias. Na Tabela 01 são mostrados esses resultados. 
Rev. Elet. em Gestão, Educação e Tecnologia Ambiental (e-ISSN: 2236-1170)

Tabela 01: Listagem dos Reagentes para as Respectivas Análises e Quantidades.

\begin{tabular}{|c|c|c|c|c|c|c|c|c|c|c|c|}
\hline & & \multicolumn{10}{|c|}{ ANÁLISES } \\
\hline Lista dos Reagentes & Unidade & DQO & Nitrito & Fósforo & N Amoniacal & Nitrato & Alcalinidade & Cloretos & Sulfato & Coliformes & Metais \\
\hline ACETATO DE SÓDIO & $\mathrm{g}$ & & & & & & & & 0,6 & & \\
\hline ÁCIDO ACÉTICO & $\mathrm{mL}$ & & & & & & & & 2,4 & & \\
\hline ÁCIDO ASCÓRBICO & g & & & 0,63 & & & & & & & \\
\hline ÁCIDO RESÓLICO & $\mathrm{g}$ & & & & & & & & & 0,1 & \\
\hline AGNO3 & $\mathrm{g}$ & & & & & & & 0,285948 & & & \\
\hline AGSO4 & $\mathrm{g}$ & 5,2 & & & & & & & & & \\
\hline ÁLCOOL ETÍLICO & $\mathrm{mL}$ & & & & 30 & & & & & & \\
\hline $\mathrm{C} 12 \mathrm{H} 8 \mathrm{~N} 2 \mathrm{H} 2 \mathrm{O}$ & $\mathrm{g}$ & 0,297 & & & & & & & & & \\
\hline CLORETO DE BÁRIO & $\mathrm{g}$ & & & & & & & & 12 & & \\
\hline CROMATO DE POTÁSSIO & $\mathrm{mL}$ & & & & & & & 6 & & & \\
\hline FENOLFTALEÍNA & g & & & & 0,5 & & & & & & \\
\hline $\mathrm{FESO} 47 \mathrm{H} 2 \mathrm{O}$ & $\mathrm{g}$ & 0,139 & & & & & & & & & \\
\hline FENH42SO4 26H2O & $\mathrm{g}$ & 34,5 & & & & & & & & & \\
\hline $\mathrm{H} 2 \mathrm{O} 2$ & $\mathrm{~mL}$ & & & & & & & 6 & & & \\
\hline $\mathrm{H} 2 \mathrm{SO} 4$ & $\mathrm{~mL}$ & 579,35 & & 1 & & & & & & & \\
\hline $\mathrm{H} 2 \mathrm{SO} 4$ & $\mathrm{~g}$ & & & 6,958 & & & 0,294 & & & & \\
\hline $\mathrm{HCl}$ & $\mathrm{mL}$ & & 2 & & & 0,083 & & & & & \\
\hline $\mathrm{HGSO} 4$ & $\mathrm{~g}$ & 8,33 & & & & & & & & & \\
\hline HNO3 & $\mathrm{mL}$ & & & 5 & & & & & & & 12,5 \\
\hline K2CR2O7 & $\mathrm{g}$ & 1,23 & & & & & & & & & \\
\hline KHPO4 & $\mathrm{g}$ & & & 0,0015 & & & & & & & \\
\hline KSBOC4H4O6 1/2H2O & g & & & 0,016 & & & & & & & \\
\hline MEIO DE CULTURA & & & & & & & & & & & \\
\hline $\mathrm{MFCH}$ & $\mathrm{g}$ & & & & & & & & & 5,2 & \\
\hline MGCL2 6H2O & $\mathrm{g}$ & & & & & & & & 3,6 & & \\
\hline MOLIBDATO DE AMÔNIA & $\mathrm{g}$ & & & 0,72 & & & & & & & \\
\hline $\mathrm{NaOH}$ & g & & & 12 & & & & & & 1 & \\
\hline NED & $g$ & & 0,02 & & & & & & & & \\
\hline $\mathrm{NH} 4 \mathrm{OH}$ & $\mathrm{mL}$ & & & & & & & 1 & & & \\
\hline NITRATO DE POTÁSSIO & $\mathrm{g}$ & & & & & & & & 0,12 & & \\
\hline SULFANILAMIDA & $\mathrm{g}$ & & 0,2 & & & & & & & & \\
\hline SULFATO DE ALUMÍNIO & $\mathrm{g}$ & & & & & & & 125 & & & \\
\hline
\end{tabular}

A Listagem para o consumo periódico dos diversos reagentes é mostrado na Tabela 02. 


\section{REGEIAUFSM}

Rev. Elet. em Gestão, Educação e Tecnologia Ambiental (e-ISSN: 2236-1170)

Tabela 02: Listagem dos Reagentes para as Respectivas Análises e Quantidades.

\begin{tabular}{|c|c|c|c|c|c|}
\hline REAGENTE & SEMANAL & MENSAL & SEMESTRAL & ANUAL & UNIDADE \\
\hline ACETATO DE SÓDIO & 0,600 & 2,4 & 14,4 & 28,8 & $\mathrm{~g}$ \\
\hline ÁCIDO ACÉTICO & 2,400 & 9,6 & 57,6 & 115,2 & $\mathrm{~mL}$ \\
\hline ÁCIDO ASCORBICO & 0,630 & 2,52 & 15,12 & 30,24 & $\mathrm{~g}$ \\
\hline ÁCIDO RESÓLICO & 0,100 & 0,4 & 2,4 & 4,8 & $\mathrm{~g}$ \\
\hline $\mathrm{AGNO}_{3}$ & 0,286 & 1,143792 & 6,862752 & 13,7255 & $\mathrm{~g}$ \\
\hline $\mathrm{AGSO}_{4}$ & 5,200 & 20,8 & 124,8 & 249,6 & $g$ \\
\hline ÁLCOOL ETÍLICO & 30,000 & 120 & 720 & 1440 & $\mathrm{~mL}$ \\
\hline $\mathrm{C}_{12} \mathrm{H}_{8} \mathrm{~N}_{2} \mathrm{H}_{2} \mathrm{O}$ & 0,297 & 1,188 & 7,128 & 14,256 & $\mathrm{~g}$ \\
\hline CLORETO DE BÁRIO & 12,000 & 48 & 288 & 576 & $\mathrm{~g}$ \\
\hline CROMATO DE POTÁSSIO & 6,000 & 24 & 144 & 288 & $\mathrm{~mL}$ \\
\hline FENOLFTALEÍNA & 0,500 & 2 & 12 & 24 & $g$ \\
\hline $\mathrm{FESO}_{4} 7 \mathrm{H}_{2} \mathrm{O}$ & 0,139 & 0,556 & 3,336 & 6,672 & $\mathrm{~g}$ \\
\hline FENH42SO4 $26 \mathrm{H} 20$ & 34,500 & 138 & 828 & 1656 & $\mathrm{~g}$ \\
\hline $\mathrm{H}_{2} \mathrm{O}_{2}$ & 6,000 & 24 & 144 & 288 & $\mathrm{~mL}$ \\
\hline $\mathrm{H}_{2} \mathrm{SO}_{4}$ & 580,350 & 2321,4 & 13928,4 & 27856,8 & $\mathrm{~mL}$ \\
\hline $\mathrm{H}_{2} \mathrm{SO}_{4}$ & 7,252 & 29,008 & 174,048 & 348,096 & g \\
\hline $\mathrm{HCL}$ & 2,083 & 8,332 & 49,992 & 99,984 & $\mathrm{~mL}$ \\
\hline $\mathrm{HGSO}_{4}$ & 8,330 & 33,32 & 199,92 & 399,84 & g \\
\hline $\mathrm{HNO} 3$ & 17,500 & 70 & 420 & 840 & $\mathrm{~mL}$ \\
\hline $\mathrm{K}_{2} \mathrm{CR}_{2} \mathrm{O}_{7}$ & 1,230 & 4,92 & 29,52 & 59,04 & $g$ \\
\hline $\mathrm{KHPO}_{4}$ & 0,002 & 0,006 & 0,036 & 0,072 & $\mathrm{~g}$ \\
\hline $\mathrm{KSBOC}_{4} \mathrm{H}_{4} \mathrm{O}_{6} 1 / 2 \mathrm{H}_{2} \mathrm{O}$ & 0,016 & 0,064 & 0,384 & 0,768 & $\mathrm{~g}$ \\
\hline MEIO DE CULTURA & 0,000 & 0 & 0 & 0 & 0 \\
\hline $\mathrm{MFCH}$ & 5,200 & 20,8 & 124,8 & 249,6 & $\mathrm{~g}$ \\
\hline $\mathrm{MgCl}_{2} 6 \mathrm{H}_{2} \mathrm{O}$ & 3,600 & 14,4 & 86,4 & 172,8 & $\mathrm{~g}$ \\
\hline MOLIBDATO DE AMONIA & 0,720 & 2,88 & 17,28 & 34,56 & g \\
\hline $\mathrm{NAOH}$ & 13,000 & 52 & 312 & 624 & $\mathrm{~g}$ \\
\hline NED & 0,020 & 0,08 & 0,48 & 0,96 & $\mathrm{~g}$ \\
\hline $\mathrm{NH}_{4} \mathrm{OH}$ & 1,000 & 4 & 24 & 48 & $\mathrm{~mL}$ \\
\hline NITRATO DE POTÁSSIO & 0,120 & 0,48 & 2,88 & 5,76 & $\mathrm{~g}$ \\
\hline SULFANILAMIDA & 0,200 & 0,8 & 4,8 & 9,6 & $g$ \\
\hline SULFATO DE ALUMÍNIO & 125,000 & 500 & 3000 & 6000 & g \\
\hline
\end{tabular}

A estimativa da deposição das substâncias químicas, em termos reais (reagentes puros) que são descartados semestralmente como rejeito das análises, tomando classificação proposta, e que são despejados na rede de esgotos, teve a seguinte ordem de valores, para sólidos e líquidos (Figura 01), respectivamente: na classe (A) foi de $3,83 \mathrm{~kg}$ e 13,4 L; na classe (B) foi de 0,3 kg e 0,5 L; 
Rev. Elet. em Gestão, Educação e Tecnologia Ambiental (e-ISSN: 2236-1170)

na classe (C) foi de 0,8 kg e 0,144 L; a classe (D) foi de 0,23 kg e 0,92 L e por fim a classe (E), que não apresentou líquidos, foi de $0,17 \mathrm{~kg}$.
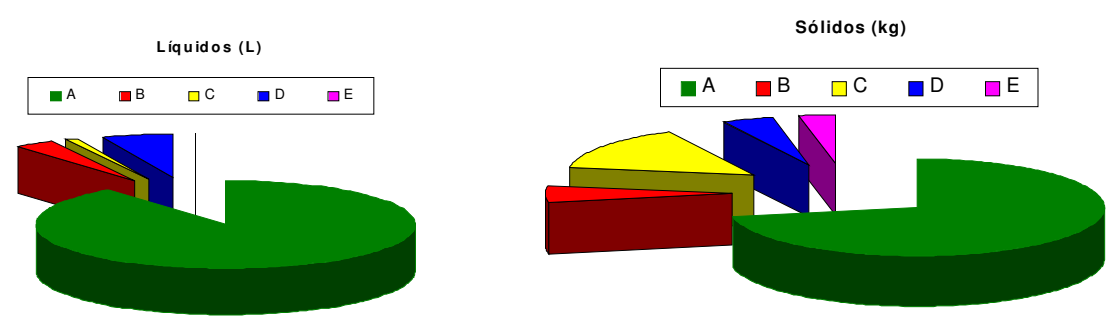

Figura 01: Resultados da Periodicidade Semestral da Geração de Resíduos

\section{CONCLUSÕES}

Dos resultados obtidos com a estimativa qualitativa e quantitativa dos resíduos do LEACQ, conclui-se que a classe majoritária, em termos de contaminação é a classe $A$, principalmente pela presença de ácido sulfúrico, reagente usado em grande escala**. Entretanto, a grande preocupação é a presença de metais pesados, classe $C$, que mesmo em pequenas quantidades, acarretam problemas em razão do acúmulo na cadeia trófica. Porém, para complementar esse trabalho se faz necessário realizar algumas análises laboratoriais para se confrontar os resultados estimados com os experimentais.

Além disso, é de fundamental importância a realização de algumas ações preventivas, como:

- Adoção de equipamentos de proteção individual;

- Conscientização e treinamento de alunos e funcionários;

- Conscientização das exigências legais e de potencial de risco de cada material presente no ambiente estudado.

A partir da caracterização realizada, será feito um estudo dos principais métodos de recuperação, como também das principais formas de destinação ambientalmente corretas para estes resíduos, levando-se em consideração, principalmente, os mais usados e os que causam maiores riscos sanitários e ambientais.

As etapas futuras seguirão das etapas de:

- Determinação de um local adequado para o armazenamento dos resíduos;

- Montagem de um sistema de coleta, armazenamento e transporte desse material;

- Escolha de recipientes, que devem combinar de forma otimizada, uma série de características (tipo, tamanho, vedação, estabilidade etc);

- Definição da estratégia de descarte, sendo nessa etapa importante o conhecimento de ações preventivas que possam diminuir o grau de risco desses resíduos (por exemplo, a neutralização de ácidos e bases).

- Criação de um programa para a redução dos resíduos e reaproveitamento por outros laboratórios ou até mesmo outras instituições. 
Rev. Elet. em Gestão, Educação e Tecnologia Ambiental (e-ISSN: 2236-1170)

\section{REFERÊNCIAS BIBLIOGRÁFICAS}

ASSOCIAÇÃO BRASILEIRA DE NORMAS TÉCNICAS. Norma NBR 12.807; Resíduos de serviço de saúde : terminologia. São Paulo, 1993.

ASSOCIAÇÃO BRASILEIRA DE NORMAS TÉCNICAS. Norma NBR 12.808; Resíduos de serviço de saúde : classificação. São Paulo, 1993.

ASSOCIAÇÃO BRASILEIRA DE NORMAS TÉCNICAS. Norma NBR 12.809; Manuseio de serviços de saúde: procedimento.. São Paulo, 1993.

ASSOCIAÇÃO BRASILEIRA DE NORMAS TÉCNICAS. Norma NBR 12.810; Coleta de serviços de saúde: procedimento.. São Paulo, 1993.

INMETRO. CTE 06; Princípios das boas práticas de Laboratório. Rio de Janeiro, SENAI.DN-NID, 1995.

INTERNATIONAL STANDARD ORGANIZATION - ISO/IEC guide 25; General requirements for the technical competence of testing laboratories 2ed. Washington, 1982.

INTERNATIONAL TECHNICAL INFORMATION INSTITUTE. Toxic and hazardous industrial chemical safety manual. Tóquio, ITI, 1977.

KLAASSEN, C.D; AMDUR, M.O.; DOULL, J. Casarett and Doull's toxicology: the basic science of poisons. 5 ed. New York, McGraw Hill, 1996.

LUNN, G. et al. Destruction of hazardous chemicals in the laboratory. New York, John Wiley \& sons, 1990.

PEDROZO, M. F. M \& PHILIPPI JR, A. Disposição de resíduos de laboratório - estudo de caso. In: 20 Congresso Brasileiro de Engenharia Sanitária e Ambiental. Rio de Janeiro RJ. Brasil. 1999. 